\section{Encephalitogenic lymphoblast recruitment to resting CNS microvasculature: a natural immunosurveillance mechanism?}

\author{
Ronen Alon \\ Department of Immunology, The Weizmann Institute of Science, Rehovot 76100, Israel. \\ Phone: 972-8-9342482; Fax: 972-8-9344141; E-mail: ronalon@wicc.weizmann.ac.il. \\ J. Clin. Invest. 108:517-519 (2001). DOI:10.1172/JCI200113646.
}

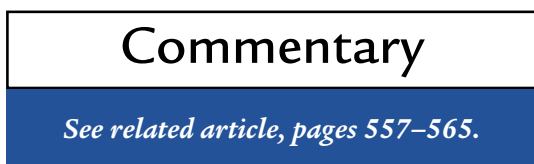

Multiple sclerosis (MS), the most common autoimmune neuronal disease, is characterized by an immunological attack against myelin in the CNS (1). Because the endothelial blood-brain barrier (BBB) tightly restricts leukocyte extravasation, the CNS is usually considered an immune-privileged site, but it is clear that autoreactive $\mathrm{T}$ cells breach this barrier in the course of this disease. Similarly, in experimental autoimmune encephalomyelitis (EAE), a disease model closely resembling MS, myelin-specific $\mathrm{CD}^{+}{ }^{+}$ T lymphocytes reach the CNS following adoptive transfer into the peripheral blood $(1,2)$.

In a previous milestone study (3), Yednock and coworkers cast light on this process with their finding that EAE induction can be prevented by administering antibodies against $\alpha 4$ integrins, such as $\alpha 4 \beta 1$ (VLA-4). In vitro, peripheral blood lymphocytes and monocytes bind inflamed but not resting brain vessels in a VLA-4-dependent manner (3). VLA-4 is a key leukocyte adhesion receptor that binds to both endothelial VCAM-1 and ECM fibronectin (4). Although myelin-specific autoreactive $\mathrm{T}$ clones must cross the CNS vasculature of healthy animals, Yednock and colleagues' results and subsequent studies (2) did not explain how autoreactive $\mathrm{T}$ blasts can infiltrate noninflamed CNS while host mononuclear cells fail to interact with CNS microvessels prior to inflammation.

Vascular beds of many extralymphoid tissues must undergo induction of specific proadhesive and stimulatory signals to recruit inflammatory effector leukocytes. Recent evidence suggests that extralymphoid vessels express basal levels of adhesive molecules, which are recognized by special- ized subsets of immune cells - potential initiators of inflammatory responses. One remarkable example is the ability of dendritic cell subsets to tether and roll on E- and P-selectins, as well as on ICAM-2, ligands that are constitutively expressed on noninflamed skin endothelium $(5,6)$ (Figure 1 ). Consequently, dendritic cells can rapidly extravasate the skin vessels upon any insult, even prior to fullblown inflammation.

Might certain encephalitogenic subsets of $\mathrm{T}$ blasts possess a similar capacity to recognize noninflamed CNS vessels? Vajkoczy et al., writing in this issue of the JCI (7), have now provided the first in vivo evidence that EAE-inducing blasts localize to and migrate through the CNS microvasculature of healthy animals. Using a novel intravital microscopy technique that allows them to view the spinal cord, these authors show that encephalitogenic $T$ blasts rely on their $\alpha 4$ integrins to arrest on the vessel wall of the CNS white matter microvasculature. Direct visualization of blasts interacting with CNS vessels, combined with mAb-blocking experiments against both blast $\alpha 4$ integrins and CNS vessel VCAM-1, shows that EAE-inducing blasts are captured by the $\mathrm{BBB}$ vessel wall primarily through VLA-4/VCAM-1 interactions (7). Once these blasts enter the CNS, if they are exposed to self antigen, they may then elicit local inflammation and thus induce other adhesive and chemotactic molecules in the nearby vascular bed. This response may thus set the stage for the entry of additional encephalitogenic lymphocytes and effector monocytes (Figure 1).

The present work (7) provides the first demonstration that the $\alpha 4$ integrin-dependence of adoptive transfer of EAE-inducing $\mathrm{T}$ blasts (3) arises from the nearly exclusive role played by the VLA-4/VCAM-1 interaction in promoting the attachment of the EAE-inducing blasts to the CNS vasculature. Both postcapillary venules and the capillary network of the CNS is suggested to express functional VCAM-1 on a constitutive basis in healthy animals (7). This VCAM-1, rather than other $\alpha 4$ integrin ligands like MadCAM-1 or the alternatively spliced fibronectin found in various endothelia (4), accounts for the capacity of $\mathrm{T}$ blasts to adhere within the microvasculature of the resting CNS (7). Generally, VCAM-1 is inducibly expressed on cytokine-activated endothelia, and therefore some of the CNS vessel expression of VCAM-1 seen in the present study may have been induced by irritation from the surgical operation. However, VCAM-1 is detected at low levels in the CNS even in healthy mice (8). The finding that anti-VCAM-1 mAb's administered to naive animals prior to or at the time of EAE induction delay onset and severity of disease $(2,9)$ also supports constitutive VCAM-1 activity on resting CNS vasculature. Despite its sparse levels, CNS-vessel VCAM-1 appears to constitute the major if not the exclusive addressin, or recruitment receptor, controlling the homing of autoreactive VLA-4-expressing $T$ blasts to the CNS. Both VLA-4 expression and affinity to VCAM-1 are higher in blast cells than in resting (naive or memory) T cells (10), potentially accounting for the inherent capacity of these blasts - but not any other leukocyte - to attach stably to endothelial VCAM-1. Indeed, VLA-4 affinity controls the ability of lymphocytes transiently tethered to 




\section{Figure 1}

A proposed model for the multistep adhesive cascade involved in the capture, arrest, and diapedesis of T blasts across resting CNS vasculature and the subsequent recruitment of mononuclear cells to the inflamed CNS. Analogous adhesive interactions that support constitutive immature dendritic cell rolling on resting skin vasculature are also shown. Antigenic stimulation in the CNS triggers both endothelial and extravascular traffic signals for subsets of effector leukocytes incapable of extravasating the resting CNS vasculature. Question marks indicate where the involvement of chemokines is still unknown.

VCAM-1-bearing surfaces to firmly capture to these surfaces under physiological shear flow (11).

In contrast to most adhesive cascades established by circulating leukocytes (12), T blast capture to the CNS vasculature does not appear to involve $\mathrm{G}_{\mathrm{i}^{-}}$ protein stimulation by endothelial chemokines (13): Blast capture to the CNS vessels is insensitive to pretreatment with pertussis toxin, a selective blocker of heterotrimeric $\mathrm{G}_{\mathrm{i}}$-protein signaling (7). However, this treatment strongly suppresses the ability of blasts to remain firmly bound to the CNS and, subsequently, to extravasate through the $\mathrm{BBB}$ endothelium (7) (Figure 1). It is therefore likely that BBB vessel chemokines, constitutively coexpressed with VCAM-1, can augment VLA-4-dependent blast adhesion on endothelial VCAM-1, and that the strengthening of this interaction represents a key step, necessary for adherent blast to transmigrate through the endothelial barrier (7). In addition, the
Vajkoczy et al. study (7) rules out the involvement of LFA-1 integrin in either blast arrest or blast migration through the CNS endothelium.

Another feature of blast associations with the CNS vasculature that sets it apart from leukocyte interactions with most endothelia is that selectins are not involved in the CNS $(7,14)$. The absence of selectins and selectin ligands on CNS vasculature renders blast $\alpha 4$ the nearly exclusive receptor responsible for blast capture to the CNS vessel wall. Although $\alpha 4$ can support leukocyte rolling on VCAM-1, both in vitro and in vivo (15), EAE-inducing blasts do not roll along the CNS vessels but rather arrest immediately after initiating contact with these vessels (7). Highaffinity $\alpha 4$, elevated on T blasts, indeed preferentially mediates leukocyte arrest on VCAM-1-bearing surfaces under shear flow $(11,16)$. This adhesive receptor thus bypasses the need for stimula-


mally essential for integrin activation and leukocyte arrest on vessel walls (13). High-affinity $\alpha 4$ also renders $T$ cells more responsive to signals by endothelial chemokines (11), potentially contributing to the $\mathrm{G}_{\mathrm{i}}$-protein-dependent migration steps that follow blast capture to CNS vessels (Figure 1). In this regard, a striking observation of the Vajkoczy et al. study is the extremely slow rates (hours) at which arrested blasts transmigrate the BBB endothelium (7). These results suggest that transendothelial migration (TEM) of blasts across the BBB is much slower than lymphocyte TEM across high endothelial venules or inflamed endothelial sites, possibly due to the existence of tight endothelial junctions in the BBB endothelium more than in other endothelial beds (17).

In addition to their specialized highaffinity binding to endothelial VCAM-1, CNS-homing blasts may be able to disrupt the tight $\mathrm{BBB}$ junctions by proteolysis. VLA-4 occupancy by VCAM-1 during lymphocyte migra- 
tion across endothelium has been shown to induce metalloprotease activity (18). It is thus possible that VLA-4 ligation by endothelial VCAM-1 augments the proteolytic potential of recruited blasts and facilitates their penetration through endothelial or subendothelial barriers.

In conclusion, the new in vivo data of Vajkoczy et al. (7) predict that VCAM- 1 is a CNS addressin with constitutive potential to recruit activated $T$ cell subsets expressing high-affinity VLA-4 to BBB vessels (Figure 1). Within the CNS microvessels, $G_{i}$ activation, perhaps induced by endotheliumderived chemokines, promotes the subsequent adhesive and migratory processes that allow the recruited $T$ blasts to extravasate through the BBB. Early CNS homing of EAE-inducing T blasts appears to be antigen-independent, since both myelin-specific and -nonspecific $\mathrm{T}$ clones migrate to a comparable degree to the CNS (19).

This apparent constitutive expression of traffic signals for autoreactive blasts on the CNS vasculature poses a paradox. Why should neuronal immune-privileged tissue express recruitment signals for self-destructive lymphocytes? The recent observation that myelin-specific autoreactive $\mathrm{T}$ clones can provide protective autoimmunity that is beneficial in CNS injuries $(20,21)$ suggests a potential solution to this enigma. $\mathrm{T}$ blasts may retain inherent capacity to home to the noninflamed CNS, thereby providing a first line of protective immunity to damaged neuronal tissue (21). MS and EAE pathogenesis may occur when this natural immunosurveillance mechanism of the CNS is deregulated.

1. Steinman, L. 1996. Multiple sclerosis: a coordinated immunological attack against myelin in the central nervous system. Cell. 85:299-302.

2. Baron, J.L., Madri, J.A., Ruddle, N.H., Hashim, G., and Janeway, C.A., Jr. 1993. Surface expression of alpha 4 integrin by CD4 $\mathrm{T}$ cells is required for their entry into brain parenchyma. J. Exp. Med. 177:57-68.

3. Yednock, T.A., et al. 1992. Prevention of experimental autoimmune encephalomyelitis by antibodies against alpha 4 beta 1 integrin. Nature. 356:63-66.

4. Lobb, R.R., and Hemler, M.E. 1994. The pathophysiologic role of alpha 4 integrins in vivo. $J$. Clin. Invest. 94:1722-1728.

5. Robert, C., et al. 1999. Interaction of dendritic cells with skin endothelium: a new perspective on immunosurveillance. J. Exp. Med. 189:627-636.

6. Geijtenbeek, T.B., et al. 2000. DC-SIGN-ICAM-2 interaction mediates dendritic cell trafficking. Nat. Immunol. 1:353-357.

7. Vajkoczy, P., Laschinger, M., and Engelhardt, B. 2001. $\alpha 4$-integrin-VCAM-1 binding mediates $G$ protein-independent capture of encephalitogenic $\mathrm{T}$ cell blasts to CNS white matter microvessels. J. Clin. Invest. 108:557-565.

8. Laschinger, M., and Engelhardt, B. 2000. Interaction of alpha4-integrin with VCAM-1 is involved in adhesion of encephalitogenic $T$ cell blasts to brain endothelium but not in their transendothelial migration in vitro. J. Neuroimmunol. 102:32-43.

9. Theien, B.E., et al. 2001. Discordant effects of anti-VLA-4 treatment before and after onset of relapsing experimental autoimmune encephalomyelitis. J. Clin. Invest. 107:995-1006.

10. Yednock, T.A., et al. 1995 . Alpha 4 beta 1 integrin- dependent cell adhesion is regulated by a low affinity receptor pool that is conformationally responsive to ligand. J. Biol. Chem. 270:28740-28750.

11. Feigelson, S.W., et al. 2001. The Src kinase p56Lck upregulates VLA-4 integrin affinity: implications for rapid spontaneous and chemokine-triggered $\mathrm{T}$ cell adhesion to VCAM-1 and fibronectin. J. Biol. Chem. 276:13891-13901.

12. Butcher, E.C., and Picker, L.J. 1996. Lymphocyte homing and homeostasis. Science. 272:60-66.

13. Campbell, J.J., and Butcher, E.C. 2000 Chemokines in tissue-specific and microenvironment-specific lymphocyte homing. Curr. Opin. Immunol. 12:336-341.

14. Engelhardt, B., Vestweber, D., Hallmann, R., and Schulz, M. 1997. E- and P-selectin are not involved in the recruitment of inflammatory cells across the blood-brain barrier in experimental autoimmune encephalomyelitis. Blood. 90:4459-4472.

15. Johnston, B., Issekutz, T.B., and Kubes, P. 1996. The alpha 4-integrin supports leukocyte rolling and adhesion in chronically inflamed postcapillary venules in vivo. J. Exp. Med. 183:1995-2006.

16. Lim, Y.C., et al. 2000. Alpha4beta1-integrin activation is necessary for high-efficiency T-cell subset interactions with VCAM-1 under flow. Microcirculation. 7:201-214.

17. Johnson-Leger, C., Aurrand-Lions, M., and Imhof, B.A. 2000. The parting of the endothelium: miracle, or simply a junctional affair? J. Cell Sci. 113:921-933.

18. Romanic, A.M., and Madri, J.A. 1994. The induction of 72-kD gelatinase in T cells upon adhesion to endothelial cells is VCAM-1 dependent. J. Cell Biol. 125:1165-1178.

19. Wekerle, H., Linington, C., Lassmann, H., and Meyermann, R. 1986. Cellular immune reactivity within the CNS. Trends Neurosci. 9:271-277.

20. Moalem, G., et al. 1999. Autoimmune T cells protect neurons from secondary degeneration after central nervous system axotomy. Nat. Med. 5:49-55

21. Schwartz, M., and Kipnis, J. 2001. Protective autoimmunity: regulation and prospects for vaccination after brain and spinal cord injuries. Trends Mol. Med. 7:252-258. 